\title{
Coherent production of hard dijets on nuclei in QCD
}

\author{
N. N. Nikolaev \\ Institut für Kernphysik (Theorie), Forschungszentrum Jülich, D-52425 Jülich, Germany \\ and L. D. Landau Institute for Theoretical Physics, Chernogolovka, Moscow Region 142 432, Russia \\ W. Schäfer and G. Schwiete \\ Institut für Kernphysik (Theorie), Forschungszentrum Jülich, D-52425 Jülich, Germany
}

(Received 5 September 2000; published 11 December 2000)

\begin{abstract}
We formulate the perturbative QCD approach to coherent diffractive dijet production in pion-nucleon and pion-nucleus collisions at high energy. For hard dijets the Pomeron splitting mechanism in which both helicity amplitudes are proportional to the unintegrated gluon structure function of the proton $\mathcal{F}\left(x, \boldsymbol{k}^{2}\right)$ and pion distribution amplitude $\phi_{\pi}(z)$ is shown to dominate. In nuclear diffraction multiple Pomeron splitting components are found to give antishadowing contributions at large jet momentum $\boldsymbol{k}$. To leading twist there is an exact cancellation of effects of nuclear attenuation and antishadowing or broadening of multiple Pomeron splitting contributions. The next-to-leading higher twist correction driven by nuclear rescatterings is calculable in hard QCD and proves to be numerically very large. We argue that large higher twist effects do not preclude the determination of gross features of $\phi_{\pi}(z)$. Our results on the atomic mass number and momentum dependence of dijet cross sections agree well with the preliminary findings from the E791 experiment.
\end{abstract}

DOI: $10.1103 /$ PhysRevD.63.014020

PACS number(s): 13.85. $-\mathrm{t}, 12.38 . \mathrm{Bx}$

\section{INTRODUCTION}

Ever since the classic work in early 1950 s by Landau, Pomeranchuk, Feinberg and Glauber on diffraction excitation of deuterons [1-3] into the proton-neutron continuum the momentum spectrum of excitation products (protons and neutrons) is known to be given by the momentum distribution of constituents in the deuteron. More recent work on diffraction dissociation focused on diffractive deep inelastic scattering (DIS). Here the microscopic QCD description of diffractive scattering by exchange of a color-singlet twogluon tower in the $t$-channel reveals a sensitivity of the mass spectrum in diffractive excitation of the continuum $q \bar{q}$ states to the gluon structure function of the target [4]. Furthermore, extending early considerations in [4] Nikolaev and Zakharov have shown in 1994 [5] that in diffraction excitation of hard dijets $\gamma^{*} p \rightarrow p^{\prime} q \bar{q}$ there exist two regimes depending on how the large transverse momentum $\boldsymbol{k}$ of the jets compares to the hard scale of DIS, i.e., whether $k \lesssim Q$ or $k \gtrsim Q$. In the first regime the transverse momentum $\boldsymbol{k}$ of jets comes from the intrinsic momentum $\boldsymbol{k}$ of the quark (antiquark) in the $q \bar{q}$ Fock state of the $\gamma^{*}$ and diffractive amplitudes are proportional to the familiar integrated gluon structure function (GSF) of the target proton $G\left(x, k^{2}\right)$, see also [6]. In the second regime, $k \gtrsim Q$, diffractive dijets are a unique probe of the differential (unintegrated) gluon structure function (DGSF) of the proton $\mathcal{F}\left(x, Q^{2}\right)=\partial G\left(x, Q^{2}\right) / \partial \log Q^{2}$. Specifically, in this regime the transverse momentum $\boldsymbol{k}$ of jets is provided not by the momentum of $q$ and $\bar{q}$ in the virtual photon, but by the momentum of gluons in the Pomeron. Correspondingly, this regime of diffractive DIS has been dubbed "the splitting of Pomerons into dijets" [5]. In this regime the diffractive dijet production amplitude is proportional to $\mathcal{F}\left(x, \boldsymbol{k}^{2}\right)$. Subsequently Golec-Biernat, Kwiecinski and Martin [7] reformulated the formalism [5] in terms of the off-diagonal (skewed) parton distributions. Because the skewed distributions can be approximated [8] by the diagonal ones at a rescaled $x$, after this rescaling the formulas of [5] are recovered.

In the present communication we extend the approach [5] to coherent diffraction of pions into dijets on the nucleon and nuclear targets. The principal novelty compared to photoand electroproduction is that the pion-quark-antiquark vertex is non-pointlike which makes splitting of the Pomeron the ever more important mechanism for hard dijets. We focus on coherent diffraction production on nuclei which has recently been measured by the E791 Collaboration [9]. The principal issues with the hard QCD interpretation of these data are whether $1.25 \leq k \leq 2.5 \mathrm{GeV}$ is sufficiently hard for the perturbation QCD (PQCD) treatment, how large are next-toleading twist corrections, what are nuclear effects and whether the extraction of the pion distribution amplitude is possible from the E791 data. The two major nuclear effects one has to deal with are nuclear attenuation and nuclear broadening of jets. The practical calculation of diffraction on nuclear targets involves evaluation of multiple gluon exchanges between nucleon and excited $q \bar{q}$ system and we take full advantage of the recent determination of the DGSF of the proton [10]. We demonstrate that the broadening of the jet momentum distribution comes entirely from the multiple Pomeron splitting diagrams. The large $\boldsymbol{k}^{2}$ behavior of $\mathcal{F}\left(x, \boldsymbol{k}^{2}\right)$ found in [10] is shown to entail a remarkable cancelation of the attenuation and broadening effects to leading twist. In view of these cancelations the principal nuclear effect is a higher twist correction which is perturbatively calculable and is proportional to $G\left(x, \boldsymbol{k}^{2}\right)$.

The further presentation is organized as follows. In Sec. II we introduce the principal formalism starting with excitation of diffractive dijets on free nucleons and isolate the two helicity components of the diffraction cross section. We demonstrate how the dominance of the Pomeron splitting mecha- 
nism into hard dijets and therefore the proportionality of diffraction amplitudes to $\mathcal{F}\left(x, \boldsymbol{k}^{2}\right)$ do emerge because of the non-pointlike pion-quark-antiquark coupling. For the same reason diffractive amplitudes are shown to be proportional to the pion lightcone distribution amplitude $\phi_{\pi}(z)$ of much discussion in the recent literature (for the review see [11-13]). The possibility of measuring $\phi_{\pi}(z)$ in diffraction of pions into hard dijets has been mentioned in [14] but as we show the claim in [14] that to the leading twist diffractive amplitudes are proportional to $G\left(x, \boldsymbol{k}^{2}\right)$ is in error. Calculation of multiple gluon exchange in diffraction off nuclei to leading and higher twist is described in Sec. III. The novel feature of nuclear diffraction are multiple-Pomeron splitting processes in which the $\boldsymbol{k}$ distribution is broadened by the gluon momentum coming from different split Pomerons. In the standard nuclear multiple-scattering expansion the higher order nuclear rescatterings are known to generate nuclear shadowing [15]. We demonstrate that after reformulation in terms of multiple Pomeron splitting components the nuclear multiple scattering expansion takes a form in which higher order Pomeron splitting components give antishadowing contributions, i.e. an enhancement of the corresponding impulse approximation term. We find an exact cancelation of effects of nuclear attenuation and antishadowing or broadening of multiple Pomeron splitting in the leading twist $\boldsymbol{k}^{2}$ distributions. The higher twist correction rises with the multiplicity of split Pomerons and is shown to be proportional to the integrated gluon structure function of the proton $G\left(x, \boldsymbol{k}^{2}\right)$. It is an antishadowing correction and rises with the nuclear mass number. In Sec. V we summarize our main results and present a comparison with the preliminary experimental findings from E791. Our numerical analysis shows that the leading plus next-to-leading twist asymptopia sets in only for $k \gtrsim 2-3$ $\mathrm{GeV}$, somewhat beyond the kinematical range of E791 $1.25 \leq k \leq 2.5 \mathrm{GeV}$. Our numerical results for the $k$ and atomic mass number dependence of the dijet cross section are consistent with the experimental findings by E791 [9].

\section{MICROSCOPIC QCD MECHANISM OF DIFFRACTION INTO DIJETS}

We only need a slight adaptation of the formalism developed in $[4,5,16]$. Diffraction dissociation of the pion into the high mass continuum, hard, $q \bar{q}$ dijet final state,

$$
\pi p \rightarrow p^{\prime} q \bar{q},
$$

is described by the four PQCD diagrams of Fig. 1. In this paper the dijet cross section is calculated at the parton level. The relevant kinematical variables are shown in Fig. $1, \Delta$ is the transverse momentum of the excited dijet, quark and antiquark jets carry a fraction $z$ and $1-z$ of the pion's momentum and the invariant mass of the excited pair $M$ is given by

$$
M^{2}=\frac{\boldsymbol{k}^{2}+m_{f}^{2}}{z(1-z)},
$$

where $m_{f}$ is the quark mass. Such a parton level modeling of final states is applicable if the invariant mass $M$ of the dif- a)

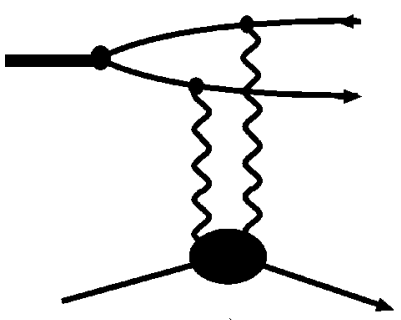

c)

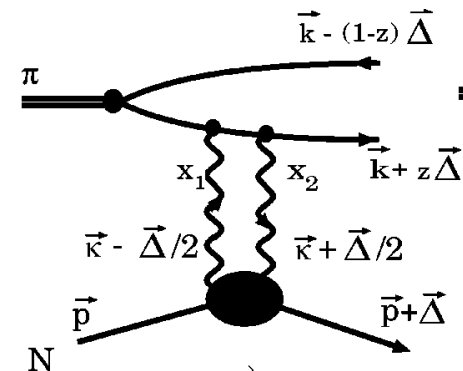

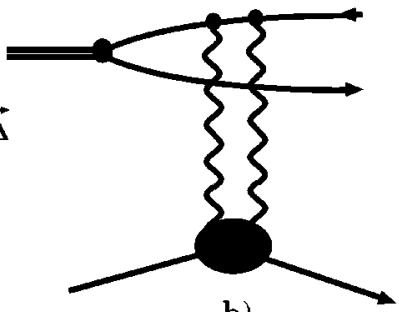

b)



d)
FIG. 1. Feynman diagrams for diffractive dijet excitation in $\pi \mathrm{N}$ collisions.

fractive system is above the prominent resonances which are excited diffractively from pions, specifically $A_{1}(1260)$, $\pi(1670), \pi(2100)$ [17] and $\pi(1300), \pi(1800)$ [18,19] and, perhaps, still higher radial and angular excitations of the pion. For instance, the color dipole model analysis in [20] has shown that diffraction excitation of nucleons is exhausted by resonance excitation for $M \lesssim 3 \mathrm{GeV}$. Therefore, the parton level calculation is viable at best for jets with $k$ $\gtrsim 1.5 \mathrm{GeV}$.

The minor technical difference from diffractive excitation of the photon studied in $[4,5,16]$ is the change from the pointlike $\gamma^{*} q \bar{q}$ vertex $e A_{\mu} \bar{\Psi} \gamma_{\mu} \Psi$ to the non-pointlike $\pi q \bar{q}$ vertex $i \Gamma_{\pi}\left(M^{2}\right) \bar{\Psi} \gamma_{5} \Psi$. In terms of the quark \& antiquark helicities $\lambda$ the $\pi \bar{q}(\boldsymbol{k}) q(-\boldsymbol{k})$ vertex has the form (for the related discussion see Jaus [21])

$$
\bar{\Psi}_{\lambda}(\boldsymbol{k}) \gamma_{5} \Psi_{\bar{\lambda}}(-\boldsymbol{k})=\frac{\lambda}{\sqrt{z(1-z)}}\left[m_{f} \delta_{\lambda-\bar{\lambda}}-\sqrt{2} \boldsymbol{k} \cdot \boldsymbol{e}_{-\lambda} \delta_{\lambda \bar{\lambda}}\right]
$$

where $m_{f}$ is the quark mass and $\boldsymbol{e}_{\lambda}=-\left(\lambda \boldsymbol{e}_{x}+i \boldsymbol{e}_{y}\right) / \sqrt{2}$. In transitions of spin-zero pions into $q \bar{q}$ states with the sum of helicities $\lambda+\bar{\lambda}= \pm 1$ the latter is compensated by the orbital angular momentum of quark and antiquark.

In close analogy to the QCD description of diffractive dijet excitation in DIS, $\gamma^{*} \rightarrow q \bar{q}$ developed in $[4,5,16]$, the two helicity transitions in Eq. (2) define the two diffractive amplitudes $\Phi_{0}(z, \boldsymbol{k}, \boldsymbol{\Delta})$ and $\boldsymbol{\Phi}_{1}(z, \boldsymbol{k}, \boldsymbol{\Delta})$. The lower blob in diagrams of Fig. 1 is related to the off-forward and offdiagonal differential gluon structure function of the target proton $\mathcal{F}\left(x_{1}, x_{2}, \boldsymbol{\kappa}, \Delta\right)$. In the considered high energy limit the two-gluon exchange interaction of $q \bar{q}$ states with the target conserves the quark and antiquark helicities exactly. This 
quark helicity conservation simplifies substantially the calculation of multiple Pomeron exchanges in diffraction off nuclei. One can readily update to the pion beam an analysis of the $\Delta$ dependence of diffractive amplitudes carried out for diffractive DIS in [23], but for the purposes of diffraction on nuclei we only need the amplitudes for $\pi N \rightarrow N(q \bar{q})$ in the forward limit $\boldsymbol{\Delta}=0$ and suppress $\boldsymbol{\Delta}$ as an argument of diffractive amplitudes wherever it is appropriate. The lightcone momentum of the gluon is related to the change of the mass of the diffractive system, $x_{g}=\left(M_{f}^{2}-M_{i n}^{2}\right) / W^{2}$. In the considered problem $M_{i n}^{2}=m_{\pi}^{2}$ can be neglected, and in the Pomeron splitting regime we have

$$
x_{1} \approx x_{\mathrm{P}}(1-z), \quad x_{2} \simeq x_{\mathrm{P}} z .
$$

The detailed discussion of skewedness is found in $[7,8]$ and need not be repeated here, the principal point is that in the diffractive limit of $x_{\mathrm{P}} \ll 1$ the relevant off-diagonal differential gluon structure function of the target proton can be approximated $[8,7]$ by the conventional DGSF taken at $x$ $=\frac{1}{2}\left(x_{1}+x_{2}\right)=\frac{1}{2} x_{\mathbb{P}}$, i.e.,

$$
\mathcal{F}\left(x_{1}, x_{2}, \boldsymbol{\kappa}, \boldsymbol{\Delta}\right)=\mathcal{F}\left(\frac{1}{2} x_{\mathrm{P}}, \boldsymbol{\kappa}, \boldsymbol{\Delta}=0\right)=\frac{\partial G\left(\frac{1}{2} x_{\mathrm{P}}, \boldsymbol{\kappa}^{2}\right)}{\partial \log \boldsymbol{\kappa}^{2}} .
$$

After this rescaling one recovers precisely the expressions of [5] for the diffractive amplitudes $\Phi_{2}, \Phi_{1}$. The hard scale in $q \bar{q}$ excitation is set by the large transverse momentum of jets, $\boldsymbol{k}^{2} \gg 1 \mathrm{GeV}^{2}$, and it is $\alpha_{S}\left(\boldsymbol{k}^{2}\right)$ which enters the gluonquark and gluon-antiquark vertices in the diffractive amplitudes.

We find it convenient to introduce

$$
\sigma_{0}=\frac{4 \pi}{3} \int d^{2} \boldsymbol{k} \frac{\mathcal{F}\left(\frac{1}{2} x_{\mathrm{P}}, \boldsymbol{k}^{2}\right)}{\boldsymbol{k}^{4}}
$$

and the distribution function

$$
f^{(1)}(\boldsymbol{k})=\frac{4 \pi}{3 \sigma_{0}} \frac{\mathcal{F}\left(\frac{1}{2} x_{\mathrm{P}}, \boldsymbol{k}^{2}\right)}{\boldsymbol{k}^{4}}
$$

normalized to unity: $\int d^{2} \boldsymbol{k} f^{(1)}(\boldsymbol{k})=1$. For the sake of brevity of notations we suppress the dependence on $x_{\mathrm{P}}$.

We define the two diffractive amplitudes $\Phi_{0}(z, \boldsymbol{k})$ and $\boldsymbol{\Phi}_{1}(z, \boldsymbol{k})$ as (we use the normalization slightly different from that in $[5,16])$

$$
\begin{aligned}
\Phi_{0}(z, \boldsymbol{k})= & \alpha_{S}\left(\boldsymbol{k}^{2}\right) \sigma_{0} \int d^{2} \boldsymbol{\kappa} m_{f} \\
& \times\left[\psi_{\pi}(z, \boldsymbol{k})-\psi_{\pi}(z, \boldsymbol{k}-\boldsymbol{\kappa})\right] f^{(1)}(\boldsymbol{\kappa}) \\
= & \alpha_{S}\left(\boldsymbol{k}^{2}\right) \sigma_{0}\left[\int d^{2} \boldsymbol{\kappa} m_{f} \psi_{\pi}(z, \boldsymbol{k}) f^{(1)}(\boldsymbol{\kappa})\right. \\
& \left.-\int d^{2} \boldsymbol{\kappa} m_{f} \psi_{\pi}(z, \boldsymbol{\kappa}) f^{(1)}(\boldsymbol{k}-\boldsymbol{\kappa})\right]
\end{aligned}
$$

$$
\begin{aligned}
\boldsymbol{\Phi}_{1}(z, \boldsymbol{k})= & \alpha_{S}\left(\boldsymbol{k}^{2}\right) \sigma_{0} \int d^{2} \boldsymbol{\kappa}\left[\boldsymbol{k} \psi_{\pi}(z, \boldsymbol{k})\right. \\
& \left.-(\boldsymbol{k}-\boldsymbol{\kappa}) \psi_{\pi}(z, \boldsymbol{k}-\boldsymbol{\kappa})\right] f^{(1)}(\boldsymbol{\kappa}) \\
= & \alpha_{S}\left(\boldsymbol{k}^{2}\right) \sigma_{0}\left[\int d^{2} \boldsymbol{\kappa} \boldsymbol{k} \psi_{\pi}(z, \boldsymbol{k}) f^{(1)}(\boldsymbol{\kappa})\right. \\
& \left.-\int d^{2} \boldsymbol{\kappa} \boldsymbol{\kappa} \psi_{\pi}(z, \boldsymbol{\kappa}) f^{(1)}(\boldsymbol{k}-\boldsymbol{\kappa})\right] .
\end{aligned}
$$

The differential cross section of forward dijet production equals

$$
\left.\frac{d \sigma_{D}}{d z d \boldsymbol{k}^{2} d \boldsymbol{\Delta}^{2}}\right|_{\boldsymbol{\Delta}=0}=\frac{\pi^{3}}{24}\left\{\left|\Phi_{0}\right|^{2}+\left|\boldsymbol{\Phi}_{1}\right|^{2}\right\} .
$$

The radial wave function of the pion in momentum space is defined in terms of the $\pi q \bar{q}$ vertex function as

$$
\psi_{\pi}(z, \boldsymbol{k})=\frac{N_{c} \Gamma_{\pi}\left(M^{2}\right)}{4 \pi^{3} z(1-z)\left(M^{2}-m_{\pi}^{2}\right)}
$$

and is so normalized that the $\pi \rightarrow \mu \nu$ decay constant equals [we use the Particle Data Group (PDG) convention $F_{\pi}$ $=131 \mathrm{MeV}[24]]$

$$
F_{\pi}=\int d^{2} \boldsymbol{k} d z m_{f} \psi_{\pi}(z, \boldsymbol{k})=F_{\pi} \int d z \boldsymbol{\phi}_{\pi}(z)
$$

Here we indicated also the relationship to the often discussed pion distribution amplitude $\phi_{\pi}(z)$ which for the purposes of our discussion we find it convenient to normalize to unity, $\int d z \phi_{\pi}(z)=1$. We recall that for the pointlike photon $\Gamma_{\gamma}\left(M^{2}\right)=e_{f}$, where $e_{f}$ is the electric charge of the quark. In contrast to the pointlike photon for the non-pointlike pion $\Gamma_{\pi}\left(M^{2}\right)$ vanishes at large $M^{2}$ faster than $\propto M^{-2}$, the relevant arguments are found in Brodsky and Lepage [13] and need not be repeated here. To this end we disagree with Ref. [14] in which the pointlike $\Gamma_{\pi}\left(M^{2}\right)=$ const is assigned to the large- $M^{2}$ tail of the pion wave function.

Let us focus on the amplitude $\Phi_{0}(z, k)$. The first term in Eq. (8) comes from diagrams $1 \mathrm{a}, 1 \mathrm{~b}$, the corresponding spectrum of jets would be identical to the quark (antiquark) momentum distribution in the pion. Because the second term is a convolution of the gluon distribution and wave function, as such it is a broader function of $\boldsymbol{k}$ than $\psi_{\pi}(z, \boldsymbol{k})$ alone and would always take over at large $\boldsymbol{k}$. The precise pattern of this dominance depends on the large- $\boldsymbol{k}$ properties of $\mathcal{F}\left(x_{\mathrm{P}}, \boldsymbol{k}^{2}\right)$, the detailed discussion of which is found in [10]. Here we only mention that for $x \sim 10^{-2}$ relevant to the E791 experiment the results of [10] correspond to the inverse power asymptotics at large- $\boldsymbol{k}^{2}$

$$
f^{(1)}(\boldsymbol{k}) \propto k^{-2 \delta}
$$




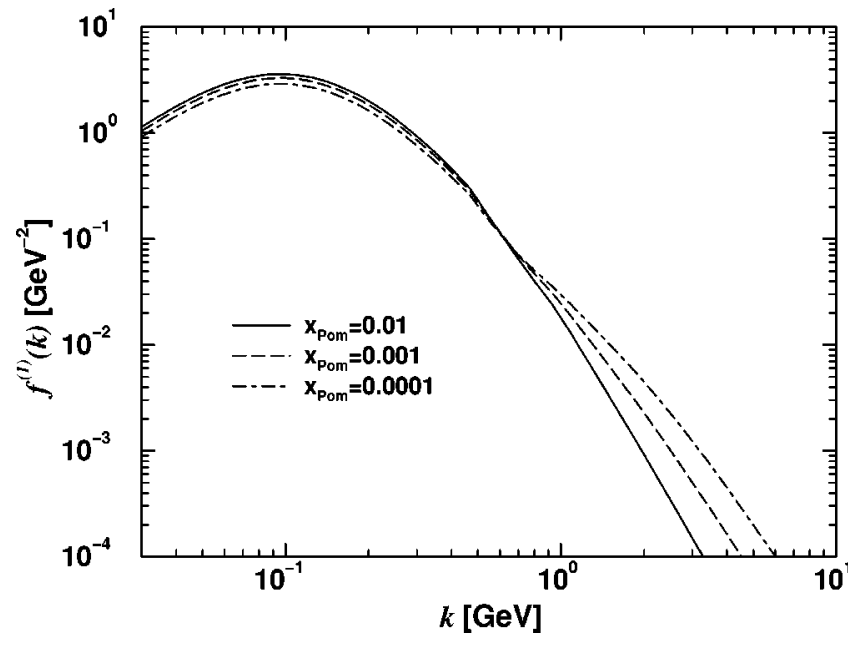

FIG. 2. The $\boldsymbol{k}$-distribution $f^{(1)}(\boldsymbol{k})$ as a function of $k$ for several values of $x_{\mathrm{P}}$.

with the exponent $\delta \sim 2.15$. At $x \sim 10^{-2}$ this asymptotics sets in at $\boldsymbol{k}^{2} \geq k_{h}^{2} \sim 1 \mathrm{GeV}^{2}$, for smaller values of $x_{\mathrm{P}}$ the exponent $\delta$ is smaller, for instance $\delta \sim 1.7$ for $x_{\mathrm{P}}=10^{-3}$, and $k_{h}^{2}$ gets larger, see Fig. 2. As such, $f^{(1)}(\boldsymbol{k})$ decreases at large $\boldsymbol{k}$ much slower than the pion wave function $\psi_{\pi}(z, \boldsymbol{k})$ [see the explicit parametrization (40) below] and the asymptotics of the convolution will be controlled by the asymptotics of $f^{(1)}(\boldsymbol{k})$. We evaluate the second term in Eq. (8) to the nextto-leading twist making use of the small- $\boldsymbol{\kappa}$ expansion

$$
f^{(1)}(\boldsymbol{k}-\boldsymbol{\kappa}) \approx f^{(1)}(\boldsymbol{k})\left(1+\frac{2 \delta \boldsymbol{k} \boldsymbol{\kappa}}{\boldsymbol{k}^{2}}-\delta \frac{\boldsymbol{\kappa}^{2}}{\boldsymbol{k}^{2}}+2 \delta(\delta+1) \frac{(\boldsymbol{k} \boldsymbol{\kappa})^{2}}{\boldsymbol{k}^{4}}\right)
$$

and obtain

$$
\begin{aligned}
\Phi_{0}(z, \boldsymbol{k})= & \alpha_{S}\left(\boldsymbol{k}^{2}\right) \sigma_{0}\left[m_{f} \psi_{\pi}(z, \boldsymbol{k})-f^{(1)}(\boldsymbol{k}) F_{\pi} \boldsymbol{\phi}_{\pi}(z)\right. \\
& \left.\times\left(1+\delta^{2} \frac{\left\langle\boldsymbol{\kappa}_{\pi}^{2}(z)\right\rangle}{\boldsymbol{k}^{2}}\right)\right],
\end{aligned}
$$

where

$$
\left\langle\boldsymbol{\kappa}_{\pi}^{2}(z)\right\rangle=\frac{\int d^{2} \boldsymbol{\kappa} \boldsymbol{\kappa}^{2} \psi_{\pi}(z, \boldsymbol{\kappa})}{\int d^{2} \boldsymbol{\kappa} \psi_{\pi}(z, \boldsymbol{\kappa})} .
$$

In the related evaluation of the large- $\boldsymbol{k}$ behavior of the convolution term in the diffractive amplitude $\boldsymbol{\Phi}_{1}$ for excitation of dijets with the sum of helicities $\lambda+\bar{\lambda}= \pm 1$ the leading term comes from the second term $\propto \delta 2 \boldsymbol{k} \boldsymbol{\kappa} / \boldsymbol{k}^{2}$ in the expansion (13). Then we find

$$
\begin{aligned}
\boldsymbol{\Phi}_{1}(z, \boldsymbol{k})= & \alpha_{S}\left(\boldsymbol{k}^{2}\right) \sigma_{0} \boldsymbol{k}\left[\psi_{\pi}(z, \boldsymbol{k})\right. \\
& \left.-\frac{\delta\left\langle\boldsymbol{\kappa}_{\pi}^{2}(z)\right\rangle}{m_{f} \boldsymbol{k}^{2}} f^{(1)}(\boldsymbol{k}) F_{\pi} \phi_{\pi}(z)\right]
\end{aligned}
$$

Evidently, in the region of large $\boldsymbol{k}$ where $\psi_{\pi}(z, \boldsymbol{k})$ dies out the amplitude $\boldsymbol{\Phi}_{1}$ will give the higher twist correction to the high- $k$ dijet cross section.

The resulting large- $\boldsymbol{k}$ asymptotics of the differential cross section for dijet production on nucleons reads

$$
\begin{aligned}
& \left.\frac{d \sigma_{D}}{d z d \boldsymbol{k}^{2} d \boldsymbol{\Delta}^{2}}\right|_{\boldsymbol{\Delta}=0} \\
& =\frac{2 \pi^{5}}{27} F_{\pi}^{2} \phi_{\pi}^{2}(z) \alpha_{S}^{2}\left(\boldsymbol{k}^{2}\right)\left[\frac{\mathcal{F}\left(\frac{1}{2} x_{\mathrm{P}}, \boldsymbol{k}\right)}{\boldsymbol{k}^{4}}\right]^{2} \cdot\left\{1+2 \delta^{2}\right. \\
& \left.\quad \times \frac{\left\langle\kappa_{\pi}^{2}(z)\right\rangle}{\boldsymbol{k}^{2}}\left(1+\frac{\left\langle\kappa_{\pi}^{2}(z)\right\rangle}{2 m_{f}^{2}}\right)\right\} .
\end{aligned}
$$

Evidently, the large- $\boldsymbol{k}$ asymptotic behavior $\propto k^{-8}$ is suggested by purely dimensional counting. Substantial departure from the law $\propto k^{-8}$ is possible because of scaling violations in $\mathcal{F}\left(\frac{1}{2} x_{\mathrm{P}}, \boldsymbol{k}\right)$. According to the recent phenomenological analysis [10], the DGSF $\mathcal{F}(x, \boldsymbol{k})$ is approximately constant at moderately small $x \sim 10^{-2}$, but rises steeply with $\boldsymbol{k}^{2}$ at $x$ $\lesssim 10^{-3}$. Also, the experimental data are taken at fixed $W^{2}$, so that in view of Eqs. (1) and (3) the $\boldsymbol{k}^{2}$ dependence of the observed cross section is affected by the increase of $x_{\mathrm{P}}$ and decrease of $\mathcal{F}\left(\frac{1}{2} x_{\mathbb{P}}, \boldsymbol{k}\right)$ with increasing $\boldsymbol{k}^{2}$. Similar kinematical bias affects the $z$-dependence of the experimentally observed cross section.

There are three important aspects of our diffractive dijet excitation amplitudes at large $\boldsymbol{k}$ where the pion wave function dies out.

First, here both helicity amplitudes are proportional to the DGSF of the target proton $\mathcal{F}\left(\frac{1}{2} x_{\mathrm{P}}, \boldsymbol{k}^{2}\right)$, i.e. The jet momentum comes from the momentum of gluons in the exchanged Pomeron, hence the term "splitting the Pomeron." To this end we recall that Nikolaev and Zakharov found the same proportionality of diffractive amplitudes to $\mathcal{F}\left(\frac{1}{2} x_{\mathrm{P}}, \boldsymbol{k}^{2}\right)$ also for real photoproduction with pointlike $\gamma q \bar{q}$ QED vertex [5]. From here one would conclude that this property does not require the wave function of the pion to be soft and the $\pi q \bar{q}$ vertex function $\Gamma_{\pi}\left(M^{2}\right)$ to vanish at large $M^{2}$. Here we disagree with [14] who claimed that diffractive amplitudes are proportional to the integrated gluon structure function $G\left(x, \boldsymbol{k}^{2}\right)$. We notice, however, that in real photoproduction the cross section is dominated by the contribution from the helicity amplitude $\boldsymbol{\Phi}_{1}$ rather than $\Phi_{0}$ in the pion case. Also, because of the pointlike $\gamma q \bar{q}$ QED vertex the photoproduction cross section is $\propto k^{-6}$, see Eq. (29) of [5], compared to $k^{-8}$ for pions as given by Eq. (17). 
Second, in the same regime of hard dijets both diffractive amplitudes are proportional to the pion decay constant $F_{\pi}$ and, more important, to the pion distribution amplitude $\phi_{\pi}(z)$. By the nature of our derivation this property emerges if the radial wave function of the pion $\psi(z, \boldsymbol{k})$ is a steeper function of $\boldsymbol{k}$ than $f^{(1)}(\boldsymbol{k})$, which holds naturally for the anticipated decrease of non-pointlike $\pi q \bar{q}$ vertex function and for the phenomenologically known gluon structure function of the proton. Consequently, the $z$-distribution of dijets allows the determination of the $z$-distribution of the pion distribution amplitude $\phi_{\pi}(z)$.

Third, we emphasize that to the leading twist the differential cross section for dijet production on nucleons [Eq. (17)] does not contain any free parameters, and thus is the perturbatively calculable quantity.

\section{NUCLEAR DIFFRACTION AMPLITUDES}

We consider coherent diffraction

$$
\pi A \rightarrow q \bar{q} A^{\prime},
$$

where the recoil nucleus $A^{\prime}$ remains in the ground state. We focus on the forward diffraction cone $\Delta^{2} \leq R_{A}^{-2}$, where $R_{A}$ is the nuclear radius. The longitudinal momentum transfer to the nucleus equals $\Delta_{z}=x_{\mathrm{P}} m_{N}$ and coherent diffraction is possible if $\Delta_{z}^{2} \ll R_{A}^{-2}$, which condition is satisfied in the E791 kinematics in which $x_{\mathrm{P}} \sim 10^{-2}$, see also the discussion in Sec. IV A.

At $x_{\mathbb{P}} \sim 10^{-2}$ nuclear effects in DIS are dominated by nuclear shadowing of the $q \bar{q}$ Fock state of the photon [2527]. Hence one must sum the $q \bar{q}$ multiple-scattering amplitudes of Fig. 3, we show a representative set for the impulse approximation, $j=1$ (Figs. 3(a), 3(b), and double scattering, $j=2$ [Figs. 3(c)-3(e)]. The typical multiplicity of rescatterings, $j$, is much smaller than the target mass number $A$. Because of the quark and antiquark helicity conservation one can calculate first the $q \bar{q}$-nucleus scattering amplitude and convolute it with the pion wave function. Because the radius of nuclei $R_{A}$ is much larger than the pion radius $R_{\pi}$ one can safely neglect the $\boldsymbol{\Delta}$ dependence coming from the $q \bar{q}$-nucleon scattering and take the $q \bar{q}$-nucleon amplitudes in the forward limit $\Delta=0$. The strong coupling enters the $q \bar{q}$ loop as $\alpha_{S}\left(\boldsymbol{k}^{2}\right)$. In the high-energy limit of $x_{\mathrm{P}} \ll 1 / R_{A} m_{N}$ the calculation and summation of nuclear multiple scattering amplitudes is readily done in the impact parameter representation $[15,28,25,26]$. Namely, we notice that after passing to the $q \bar{q}$ color dipole representation the helicity amplitudes $\Phi_{0}(z, \boldsymbol{k})$ and $\boldsymbol{\Phi}_{1}(z, \boldsymbol{k})$ can be cast in the form

$$
\begin{aligned}
& \Phi_{0}(z, \boldsymbol{k})=\int d^{2} \boldsymbol{r} e^{-i \mathbf{k} \boldsymbol{r}} \sigma(x, \boldsymbol{r}) m_{f} \Psi_{\pi}(\boldsymbol{r}, z) \\
& \boldsymbol{\Phi}_{1}(z, \boldsymbol{k})=-i \int d^{2} \boldsymbol{r} e^{-i \boldsymbol{k} \mathbf{r}} \sigma(x, \boldsymbol{r}) \boldsymbol{\nabla} \Psi_{\pi}(\boldsymbol{r}, z) .
\end{aligned}
$$

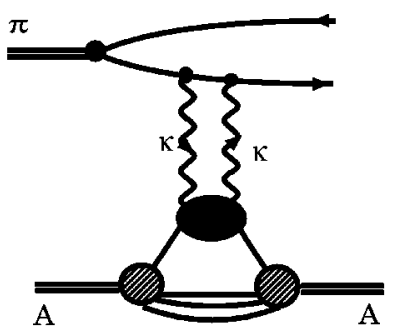

a)

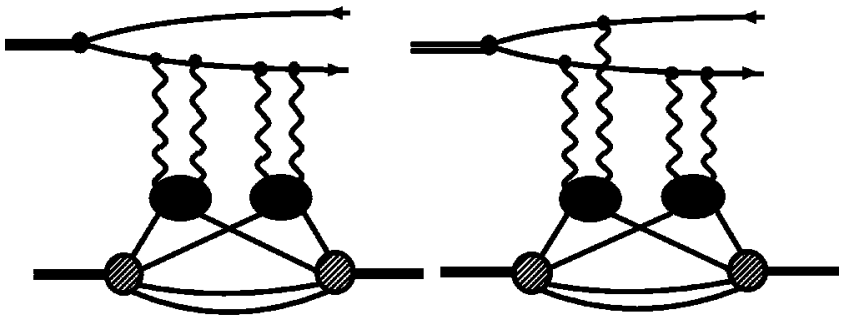

c)

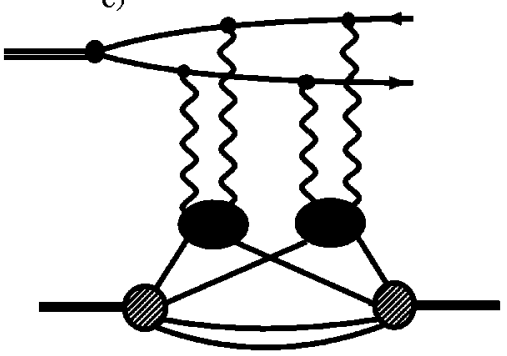

e)
FIG. 3. The nuclear multiple scattering series for diffractive dijet excitation on nuclei. Diagrams (a),(b) are sample diagrams of impulse approximation, diagrams (c)-(e) represent the various types of double scattering contributions. Higher order contributions, that appear in the calculation are not shown.

Here $\boldsymbol{k}$ is the transverse momentum of the jet, $\boldsymbol{r}$ is the $q \bar{q}$ separation in the impact parameter plane,

$$
\Psi_{\pi}(z, \boldsymbol{r})=\frac{1}{(2 \pi)^{2}} \int d^{2} \boldsymbol{k} \psi_{\pi}(z, \boldsymbol{k}) \exp (i \boldsymbol{k r})
$$

is proportional to the $q \bar{q}$ color dipole distribution amplitude in the pion and

$$
\sigma(x, \boldsymbol{r})=\alpha_{S}\left(\boldsymbol{k}^{2}\right) \sigma_{0} \int d^{2} \boldsymbol{\kappa} f^{(1)}(\boldsymbol{\kappa})[1-\exp (i \boldsymbol{\kappa} \boldsymbol{r})]
$$

has the meaning of the dipole cross section for interaction of the $q \bar{q}$ dipole $\boldsymbol{r}$ with the target nucleon in which the strong coupling $\alpha_{S}$ enters at the hard scale given by the jet transverse momentum $\boldsymbol{k}$.

The Glauber-Gribov representation of amplitudes (18) for the nuclear target is obtained by substitution of the $q \bar{q}$-nucleon scattering amplitude by the $q \bar{q}$-nucleus scattering amplitude $[28,25,26]$ and reads 


$$
\begin{aligned}
\Phi_{0}^{(A)}(z, \boldsymbol{k}, \boldsymbol{\Delta})= & 2 m_{f} \int d^{2} \boldsymbol{b} \int d^{2} \boldsymbol{r} e^{-i \boldsymbol{b} \boldsymbol{\Delta}-i \boldsymbol{k} \mathbf{r}} \Psi_{\pi}(z, \boldsymbol{r}) \\
& \left.\times\left\{1-\exp -\frac{1}{2} \sigma(x, \boldsymbol{r}) T_{A}(\boldsymbol{b})\right]\right\} \\
= & 2 m_{f} \int d^{2} \boldsymbol{b} \int d^{2} \boldsymbol{r} e^{-i \boldsymbol{b} \boldsymbol{\Delta}-i \boldsymbol{k} \mathbf{r}} \Psi_{\pi}(z, \boldsymbol{r}) \\
& \times \sum_{n \geqslant 1}(-1)^{n+1} \frac{\sigma^{n}(x, \mathbf{r})}{2^{n} n !} T_{A}^{n}(\boldsymbol{b}), \\
\boldsymbol{\Phi}_{1}^{(A)}(z, \boldsymbol{k}, \boldsymbol{\Delta})= & -2 i \int d^{2} \boldsymbol{b} \int d^{2} \boldsymbol{r} e^{-i \boldsymbol{b} \boldsymbol{\Delta}-i \boldsymbol{k} \mathbf{r}} \\
& \times\left[\sum_{n \geqslant 1}(-1)^{n+1} \frac{\sigma^{n}(x, \boldsymbol{r})}{2^{n} n !} T_{A}^{n}(\boldsymbol{b})\right] \\
& \times \boldsymbol{\nabla} \Psi_{\pi}(z, \boldsymbol{r}),
\end{aligned}
$$

where $\boldsymbol{b}$ is the pion-nucleus impact parameter, $T_{A}(\boldsymbol{b})$ $=\int d z^{\prime} n_{A}\left(\boldsymbol{b}, z^{\prime}\right)$ is the familiar nuclear optical thickness [15], $n_{A}\left(\boldsymbol{b}, z^{\prime}\right)$ is the nuclear matter density. The frozen dipole approximation (21) is applicable because $\Delta_{z}^{2} \ll R_{A}^{-2}$
([28], for the modern formalism see [26]). For the sake of simplicity above we took the exponentiated form for the nuclear profile function instead of its more exact form

$$
\begin{aligned}
\Gamma_{A}(\boldsymbol{b}) & =1-\left[1-\frac{\sigma(x, \boldsymbol{r}) T_{A}(\boldsymbol{b})}{2 A}\right]^{A} \\
& \simeq\left[1-\exp \left[-\frac{1}{2} \sigma(x, \boldsymbol{r}) T_{A}(\boldsymbol{b})\right]\right],
\end{aligned}
$$

reformulation of all results for the polynomial form poses no problems.

Representative diagrams for the impulse approximation, $j=1$, are shown in Figs. 3(a), 3(b). They give the familiar result

$$
\begin{aligned}
\Phi_{0}^{(A)}(z, \boldsymbol{k}, \boldsymbol{\Delta}) & =\Phi_{0}(z, \boldsymbol{k}) \int d^{2} \boldsymbol{b} e^{-i \boldsymbol{b} \boldsymbol{\Delta}} T_{A}(\boldsymbol{b}) \\
& =A \Phi_{0}(z, \boldsymbol{k}) G_{e m}(\boldsymbol{\Delta})
\end{aligned}
$$

where $G_{e m}(\Delta)$ is the charge form factor of the nucleus.

The principal effect of rescattering is readily seen from the double scattering, $j=2$. Making use of the integral representation (20) we find

$$
\begin{aligned}
\int d^{2} \boldsymbol{r} e^{-i k r} \Psi_{\pi}(\boldsymbol{r}, z) \sigma^{2}(x, \boldsymbol{r}) & =\alpha_{S}^{2}\left(\boldsymbol{k}^{2}\right) \sigma_{0}^{2} \int d^{2} \boldsymbol{\kappa}_{1} d^{2} \boldsymbol{\kappa}_{2} f^{(1)}\left(\boldsymbol{\kappa}_{1}\right) f^{(1)}\left(\boldsymbol{\kappa}_{2}\right) \int d^{2} \boldsymbol{r} e^{-i \boldsymbol{k} r}\left[1-e^{i \boldsymbol{\kappa}_{1} \boldsymbol{r}}-e^{i \boldsymbol{\kappa}_{2} \boldsymbol{r}}+e^{i\left(\boldsymbol{\kappa}_{1}+\boldsymbol{\kappa}_{2}\right) \boldsymbol{r}}\right] \Psi_{\pi}(\boldsymbol{r}, z) \\
& =\alpha_{S}^{2}\left(\boldsymbol{k}^{2}\right) \sigma_{0}^{2} \int d^{2} \boldsymbol{\kappa}_{1} d^{2} \boldsymbol{\kappa}_{2} f^{(1)}\left(\boldsymbol{\kappa}_{1}\right) f^{(1)}\left(\boldsymbol{\kappa}_{2}\right)\left[\Psi_{\pi}(\boldsymbol{k}, z)-2 \Psi_{\pi}\left(\boldsymbol{k}-\boldsymbol{\kappa}_{1}, z\right)+\Psi_{\pi}\left(\boldsymbol{k}-\boldsymbol{\kappa}_{1}-\boldsymbol{\kappa}_{2}, z\right)\right] \\
& =\alpha_{S}^{2}\left(\boldsymbol{k}^{2}\right) \sigma_{0}^{2}\left[\Psi_{\pi}(\boldsymbol{k}, z)-2 \int d^{2} \boldsymbol{\kappa}_{\pi} \Psi_{\pi}(\boldsymbol{\kappa}, z) f^{(1)}(\boldsymbol{k}-\boldsymbol{\kappa})+\int d^{2} \boldsymbol{\kappa}_{\pi}(\boldsymbol{\kappa}, z) f^{(2)}(\boldsymbol{k}-\boldsymbol{\kappa})\right]
\end{aligned}
$$

where

$$
f^{(2)}(\boldsymbol{k})=\int d^{2} \boldsymbol{\kappa}_{1} d^{2} \boldsymbol{\kappa}_{2} f^{(1)}\left(\boldsymbol{\kappa}_{1}\right) f^{(1)}\left(\boldsymbol{\kappa}_{2}\right) \delta\left(\boldsymbol{k}-\boldsymbol{\kappa}_{1}-\boldsymbol{\kappa}_{2}\right)
$$

is normalized to unity: $\int d^{2} \boldsymbol{k} f^{(2)}(\boldsymbol{k})=1$. The sum of the impulse approximation, $n=1$, and double-scattering terms equals (for the sake of illustration we take $\Delta=0$ )

$$
\begin{aligned}
\Phi_{0}^{(A)}(z, \boldsymbol{k}, \boldsymbol{\Delta}=0)= & \alpha_{S}\left(\boldsymbol{k}^{2}\right) \sigma_{0} m_{f} \int d^{2} \boldsymbol{b} T_{A}(\boldsymbol{b})\left\{\psi_{\pi}(z, \boldsymbol{k})\left[1-\frac{1}{2} \alpha_{S}\left(\boldsymbol{k}^{2}\right) \sigma_{0} T_{A}(\boldsymbol{b})\right]-\int d^{2} \boldsymbol{\kappa} \psi_{\pi}(z, \boldsymbol{\kappa}) f^{(1)}(\boldsymbol{k}-\boldsymbol{\kappa})\right. \\
& \left.\times\left[1-\frac{1}{2} \alpha_{S}\left(\boldsymbol{k}^{2}\right) \sigma_{0} T_{A}(\boldsymbol{b})\right]-\frac{1}{2} \int d^{2} \boldsymbol{\kappa} \psi_{\pi}(z, \boldsymbol{\kappa}) f^{(2)}(\boldsymbol{k}-\boldsymbol{\kappa}) \alpha_{S}\left(\boldsymbol{k}^{2}\right) \sigma_{0} T_{A}(\boldsymbol{b})\right\} .
\end{aligned}
$$

The three terms in the last line of Eqs. (24) and (26) correspond to the three classes of double scattering diagrams shown in Figs. 3(c)-3(e). The first term in the right-hand side (RHS) of Eq. (26) shows that the no-Pomeron splitting term in Eq. (12) coming from Fig. 3(a) in the nuclear case receives the conventional shadowing correction from the double-scattering diagram of Fig. 3(c) (and the not shown here partner diagram in which the two gluons from second nucleon couple to the antiquark). The Pomeron splitting term in Eq. (12) coming from Fig. 3(b) in the nuclear case is similarly shadowed by double-scattering diagrams of Fig. 3(d). The effective shadowing cross section equals

$$
\sigma_{e f f}\left(\boldsymbol{k}^{2}\right)=\alpha_{S}\left(\boldsymbol{k}^{2}\right) \sigma_{0}
$$

The new feature of double scattering is the third term in Eq. (26) given by the double-Pomeron splitting diagram of Fig. 3(e). The convolution (25) implies the broadening of $f^{(2)}(\boldsymbol{k})$ compared to $f^{(1)}(\boldsymbol{k})$. Furthermore, this broadened distribution $f^{(2)}(\boldsymbol{k})$ has the same sign as, i.e. it is an antishadowing correction to, the single-Pomeron splitting term (26) and would eventually take 
over single-Pomeron splitting at large $\boldsymbol{k}$.

Higher order rescatterings give rise to distributions with $j$-fold Pomeron splitting

$$
f^{(j)}(\boldsymbol{k})=\int d^{2} \boldsymbol{\kappa}_{1} \ldots d^{2} \boldsymbol{\kappa}_{j} f^{(1)}\left(\boldsymbol{\kappa}_{1}\right) f^{(1)}\left(\boldsymbol{\kappa}_{2}\right) \ldots f^{(1)}\left(\boldsymbol{\kappa}_{j}\right) \delta\left(\boldsymbol{k}-\sum_{i=1}^{j} \boldsymbol{\kappa}_{i}\right)
$$

which obviously broaden with increasing $j$. Rearranging nuclear diffractive amplitudes as an expansion over $f^{(j)}(\boldsymbol{k})$ we obtain

$$
\begin{aligned}
& \Phi_{0}^{(A)}(z, \boldsymbol{k})=2 m_{f} \sum_{j \geqslant 1} \int d^{2} \boldsymbol{b} e^{-i \boldsymbol{b} \boldsymbol{\Delta}} \int d^{2} \boldsymbol{\kappa}\left[\Psi_{\pi}(z, \boldsymbol{k})-\Psi_{\pi}(z, \boldsymbol{k}-\boldsymbol{\kappa})\right] f^{(j)}(\boldsymbol{\kappa}) \frac{1}{j !}\left[\frac{\sigma_{e f f}\left(\boldsymbol{k}^{2}\right) T_{A}(\boldsymbol{b})}{2}\right]^{j} \exp \left[-\frac{\sigma_{e f f}\left(\boldsymbol{k}^{2}\right)}{2} T_{A}(\boldsymbol{b})\right] \\
& =2 m_{f} \int d^{2} \boldsymbol{b} e^{-i \boldsymbol{b} \boldsymbol{\Delta}}\left\{\Psi_{\pi}(z, \boldsymbol{k})\left[1-\exp \left(-\frac{\sigma_{e f f}\left(\boldsymbol{k}^{2}\right)}{2} T_{A}(\boldsymbol{b})\right)\right]\right. \\
& \left.-\sum_{j \geqslant 1} \int d^{2} \boldsymbol{\kappa} \Psi_{\pi}(z, \boldsymbol{\kappa}) f^{(j)}(\boldsymbol{k}-\boldsymbol{\kappa}) \frac{1}{j !}\left[\frac{\sigma_{e f f}\left(\boldsymbol{k}^{2}\right) T_{A}(\boldsymbol{b})}{2}\right]^{j} \exp \left[-\frac{\sigma_{e f f}\left(\boldsymbol{k}^{2}\right)}{2} T_{A}(\boldsymbol{b})\right]\right\}, \\
& \boldsymbol{\Phi}_{1}^{(A)}(z, \boldsymbol{k})=2 \sum_{j \geqslant 1} \int d^{2} \boldsymbol{b} e^{-i \boldsymbol{b} \boldsymbol{\Delta}} \int d^{2} \boldsymbol{\kappa}\left[\boldsymbol{k} \Psi_{\pi}(z, \boldsymbol{k})-(\boldsymbol{k}-\boldsymbol{\kappa}) \Psi_{\pi}(z, \boldsymbol{k}-\boldsymbol{\kappa})\right] f^{(j)}(\boldsymbol{\kappa}) \frac{1}{j !}\left[\frac{\sigma_{e f f}\left(\boldsymbol{k}^{2}\right) T_{A}(\boldsymbol{b})}{2}\right]^{j} \exp \left[-\frac{\sigma_{e f f}\left(\boldsymbol{k}^{2}\right)}{2} T_{A}(\boldsymbol{b})\right] \\
& =2 \int d^{2} \boldsymbol{b} e^{-i \boldsymbol{b} \Delta}\left\{\boldsymbol{k} \Psi_{\pi}(z, \boldsymbol{k})\left[1-\exp \left(-\frac{\sigma_{e f f}\left(\boldsymbol{k}^{2}\right)}{2} T_{A}(\boldsymbol{b})\right)\right]\right. \\
& \left.-\sum_{j \geqslant 1} \int d^{2} \boldsymbol{\kappa} \boldsymbol{\kappa} \Psi_{\pi}(z, \boldsymbol{\kappa}) f^{(j)}(\boldsymbol{k}-\boldsymbol{\kappa}) \frac{1}{j !}\left[\frac{\sigma_{e f f}\left(\boldsymbol{k}^{2}\right) T_{A}(\boldsymbol{b})}{2}\right]^{j} \exp \left[-\frac{\sigma_{e f f}\left(\boldsymbol{k}^{2}\right)}{2} T_{A}(\boldsymbol{b})\right]\right\} \text {. }
\end{aligned}
$$

The nuclear attenuation factors show that shadowing is indeed controlled by $\sigma_{e f f}\left(\boldsymbol{k}^{2}\right)$. Despite the decrease $\sigma_{e f f}\left(\boldsymbol{k}^{2}\right)$ $\propto \alpha_{S}\left(\boldsymbol{k}^{2}\right)$ numerically this cross section is quite large, grows slowly at very small $x_{\mathrm{P}}$, and is a soft gluon exchange dominated quantity.

At large jet momentum the diffractive amplitude is dominated by the second term in Eq. (29) in which all broadened $j$-Pomeron splitting contributions enter remarkably with the same antishadowing sign.

Whether this antishadowing takes over shadowing depends on the large $\boldsymbol{k}^{2}$ behavior of $f^{(2)}(\boldsymbol{k})$. For the power asymptotics (12) the leading contribution to convolution $f^{(2)}(\boldsymbol{k})(25)$ at large $\boldsymbol{k}^{2}$ comes from the configurations when there is one hard splitting of the Pomeron with $\kappa_{i} \sim \boldsymbol{k}$, whereas all other $\kappa_{i}$ are small. Correspondingly, the values of gluon lightcone momenta $x_{i}$ in the hard splitting of the Pomeron are the same as in the free nucleon case, whereas in the predominantly soft rescatterings $x_{i} \sim x_{S} \sim 1 \mathrm{GeV}^{2} / W^{2}$ and in multiple scattering expansion (29) all $\sigma_{e f f}\left(\boldsymbol{k}^{2}\right)$ but one for the hard splitting of the Pomeron must arguably be evaluated at $x=x_{S}$. However, we notice that according to [10] the $x$-dependence of $\mathcal{F}\left(x, \boldsymbol{k}^{2}\right)$ is weak for soft $\boldsymbol{k}^{2}$ and in the practical evaluation of $f^{(j)}(\boldsymbol{k})$ one can put $x_{S}=x_{\mathrm{P}} / 2$. Then to the leading twist one readily finds the large- $\boldsymbol{k}^{2}$ asymptotics

$$
f^{(j)}(\boldsymbol{k})=j f^{(1)}(\boldsymbol{k})
$$

which clearly shows the anticipated enhancement of the large- $\boldsymbol{k}^{2}$ tail by multiple rescatterings. The salient feature of large- $\boldsymbol{k}^{2}$ broadening (30) is that the exponent of the power asymptotics is sustained. Because of the normalization condition $\int d^{2} \boldsymbol{k} f^{(j)}(\boldsymbol{k})=1$ the broadening at large $\boldsymbol{k}$ entails the small- $\boldsymbol{k}$ depletion $f^{(j \geqslant 2)}(\boldsymbol{k})<f^{(1)}(\boldsymbol{k})$ at small- $\boldsymbol{k}$. Evidently, for larger $j$ this small- $\boldsymbol{k}$ depletion will be stronger and would extend to larger $k$.

The evaluation of the higher twist correction making use of the expansion (14) proceeds as follows. For the sake of definiteness focus on the contribution from the configuration in which $\boldsymbol{\kappa}_{1} \approx \boldsymbol{k}$ and the total transverse momentum for the subset $i=2, \ldots, j$ is small, $\left(\sum_{i \geqslant 2} \boldsymbol{\kappa}_{i}\right)^{2} \lesssim \boldsymbol{k}^{2}$. Then, to the leading $\log k^{2}$ accuracy the higher twist contribution will be dominated by the $(j-1)$ configurations of the subset $i$ $=2, \ldots, j$ in which one of the $\boldsymbol{\kappa}_{i}^{2}$ is running up to $\sim \boldsymbol{k}^{2}$ whereas all other momenta are small:

$$
\begin{aligned}
f^{(j)}(\boldsymbol{k}) \simeq & j f^{(1)}(\boldsymbol{k}) \int d^{2} \boldsymbol{\kappa}_{2} \ldots d^{2} \boldsymbol{\kappa}_{j} \\
& \times\left[1+\frac{\delta^{2}}{\boldsymbol{k}^{2}}\left(\sum_{i \geqslant 2} \boldsymbol{\kappa}_{i}\right)^{2}\right] f^{(1)}\left(\boldsymbol{\kappa}_{2}\right) \ldots f^{(1)}\left(\boldsymbol{\kappa}_{j}\right) \\
= & j f^{(1)}(\boldsymbol{k})\left[1+\frac{\delta^{2}}{\boldsymbol{k}^{2}}(j-1) \int^{\boldsymbol{k}^{2}} d^{2} \boldsymbol{\kappa}^{2} f^{(1)}(\boldsymbol{\kappa})\right] \\
= & j f^{(1)}(\boldsymbol{k})\left[1+\frac{4 \pi^{2} \delta^{2}}{3 \sigma_{0} \boldsymbol{k}^{2}}(j-1) G\left(\frac{1}{2} x_{\mathrm{P}}, \boldsymbol{k}^{2}\right)\right]
\end{aligned}
$$

Remarkably, the coefficient of the higher twist correction is proportional to the gluon structure function of the proton 
$G\left(\frac{1}{2} x_{\mathrm{P}}, \boldsymbol{k}^{2}\right)$ at a hard scale $\boldsymbol{k}^{2}$. For the $j$-Pomeron splitting diagrams the higher twist correction rises $\propto(j-1)$, i.e. The antishadowing or broadening of large- $k^{2}$ tail takes place also to the higher twist.

Making use of the result (31) in the expansion (29) we obtain

$$
\begin{aligned}
\Phi_{0}^{(A)}(z, \boldsymbol{k}, \boldsymbol{\Delta})= & 2 \int d^{2} \boldsymbol{b} e^{-i \boldsymbol{b} \boldsymbol{\Delta}}\left\{m_{f} \Psi_{\pi}(\boldsymbol{k}, z)\right. \\
& \times\left[1-\exp \left(-\frac{1}{2} \sigma_{e f f}\left(\boldsymbol{k}^{2}\right) T_{A}(\boldsymbol{b})\right)\right] \\
& -\frac{1}{2} f^{(1)}(\boldsymbol{k}) F_{\pi} \phi_{\pi}(z) \sigma_{0} \alpha_{S}\left(\boldsymbol{k}^{2}\right)\left[T_{A}(\boldsymbol{b})\right. \\
& \left.\left.+\frac{2 \pi^{2} \delta^{2} \alpha_{S}\left(\boldsymbol{k}^{2}\right)}{3 \boldsymbol{k}^{2}} G\left(\boldsymbol{k}^{2}\right) T_{A}^{2}(\boldsymbol{b})\right]\right\} .
\end{aligned}
$$

Here the impulse approximation term $\propto \Psi_{\pi}(k, z)$ is shadowed with the soft cross section (27). The most remarkable feature of the Pomeron splitting contributions (32) is an exact cancelation of soft shadowing and antishadowing or broadening effects. Furthermore, this cancelation makes redundant the exact value of $x_{S}$ at which $\sigma_{0}$ must be taken in the nuclear multiple scattering expansion. The broadening law (30) is crucial for this cancelation of soft shadowing and antishadowing/broadening effects. Similar exact cancelation of shadowing and antishadowing/broadening effects, and independence on the exact value of soft shadowing cross section $\sigma_{0}$, take place in the next-to-leading twist correction too. Consequently, both leading and next-to-leading twist amplitudes are parameter free calculable in hard perturbative QCD.

Following the derivation (16) for the free nucleon target, a similar analysis can be repeated for the nuclear target with the result

$$
\begin{array}{rl}
\boldsymbol{\Phi}_{1}^{(A)}(z, \boldsymbol{k}, \boldsymbol{\Delta}) \\
=2 & \boldsymbol{k} \int d^{2} \boldsymbol{b} e^{-i \boldsymbol{b} \boldsymbol{\Delta}}\left\{\Psi_{\pi}(\boldsymbol{k}, z)\right. \\
& \times\left[1-\exp \left(-\frac{1}{2} \sigma_{e f f}\left(\boldsymbol{k}^{2}\right) T_{A}(\boldsymbol{b})\right)\right] \\
& \left.-\frac{1}{2} \sigma_{0} \alpha_{S}\left(\boldsymbol{k}^{2}\right) \frac{\delta\left\langle\boldsymbol{\kappa}_{\pi}^{2}(z)\right\rangle}{m_{f} \boldsymbol{k}^{2}} f^{(1)}(\boldsymbol{k}) F_{\pi} \phi_{\pi}(z) T_{A}(\boldsymbol{b})\right\},
\end{array}
$$

where we neglect the corrections $\propto k^{-2}$ to the already higher twist convolution term.

We notice that within the diffraction cone of $\Delta^{2} \leqslant R_{A}^{2}$ the viable approximation is

$$
\int d^{2} \boldsymbol{b} e^{-i b \boldsymbol{\Delta}} T_{A}^{2}(\boldsymbol{b})=\frac{3 C_{A} A^{2}}{4 \pi\left\langle R_{c h}^{2}\right\rangle} G_{e m}\left(\frac{1}{2} \boldsymbol{\Delta}^{2}\right),
$$

where the coefficient $C_{A} \approx 1$ depends slightly on the shape of the nuclear matter distribution: $C_{A}=1$ for the Gaussian density appropriate for light nuclei decreases slowly to $C_{A}=\frac{9}{10}$ for the uniform density sphere. Then for large jet momentum $\boldsymbol{k}$ when the impulse approximation contribution dies out, our nuclear diffractive amplitudes (32), (33) take a particularly simple form

$$
\begin{aligned}
\Phi_{0}^{(A)}(z, \boldsymbol{k}, \boldsymbol{\Delta})= & -A F_{\pi} \phi_{\pi}(z) \frac{4 \pi \alpha_{S}\left(\boldsymbol{k}^{2}\right)}{3} \frac{\mathcal{F}\left(\frac{1}{2} x_{\mathrm{P}}, \boldsymbol{k}^{2}\right)}{\boldsymbol{k}^{4}} \\
& \times\left(1+\delta^{2} \frac{\left\langle\kappa_{\pi}^{2}(z)\right\rangle}{\boldsymbol{k}^{2}}\right) \\
& \times\left\{G_{e m}\left(\boldsymbol{\Delta}^{2}\right)+\frac{\pi \delta^{2} C_{A} A \alpha_{S}\left(\boldsymbol{k}^{2}\right)}{2\left\langle R_{c h}^{2}\right\rangle \boldsymbol{k}^{2}}\right. \\
& \left.\times G\left(\frac{1}{2} x_{\mathrm{P}}, \boldsymbol{k}^{2}\right) G_{e m}\left(\frac{1}{2} \Delta^{2}\right)\right\}, \\
\boldsymbol{\Phi}_{1}^{(A)}(z, \boldsymbol{k}, \boldsymbol{\Delta})= & -\boldsymbol{k} A F_{\pi} \phi_{\pi}(z) \frac{4 \pi \alpha_{S}\left(\boldsymbol{k}^{2}\right)}{3} \frac{\mathcal{F}\left(\frac{1}{2} x_{\mathrm{P}}, \boldsymbol{k}^{2}\right)}{\boldsymbol{k}^{4}} \\
& \times G_{e m}\left(\boldsymbol{\Delta}^{2}\right) \frac{\delta\left\langle\kappa_{\pi}^{2}(z)\right\rangle}{m_{f} \boldsymbol{k}^{2}} .
\end{aligned}
$$

Finally, a correction for the finite longitudinal momentum transfer to the nucleus can be evaluated as follows. First, within the diffraction cone the impulse approximation amplitude (23) acquires the longitudinal form factor $G_{e m}\left(x_{\mathrm{P}}^{2} m_{N}^{2}\right)$. Second, we have shown that in multiple rescattering contributions there is only one hard splitting of the Pomeron with the longitudinal momentum transfer $\Delta_{z} \approx x_{\mathbb{P}} m_{N}$ whereas in soft rescatterings the longitudinal momentum transfer can be neglected. As a result, the same longitudinal form factor $G_{e m}\left(x_{\mathrm{P}}^{2} m_{N}^{2}\right)$ holds for all multiple rescatterings.

Upon the $\Delta^{2}$ integration we find that the large- $\boldsymbol{k}^{2}$ asymptotics of nuclear diffraction cross section will read

$$
\begin{aligned}
\frac{d \sigma_{D}}{d z d \boldsymbol{k}^{2}}= & \frac{2 \pi^{5}}{27} F_{\pi}^{2} \phi_{p i}^{2}(z) G_{e m}^{2}\left(x_{\mathrm{P}}^{2} m_{N}^{2}\right) \alpha_{S}^{2}\left(\boldsymbol{k}^{2}\right) \\
& \times\left[\frac { \mathcal { F } ( \frac { 1 } { 2 } x _ { \mathrm { P } } , \boldsymbol { k } ^ { 2 } ) ] ^ { 2 } } { \boldsymbol { k } ^ { 4 } } \cdot \frac { 3 A ^ { 2 } } { \langle R _ { c h } ^ { 2 } \rangle } \cdot \left\{1+\frac{\delta^{2}}{\boldsymbol{k}^{2}}\right.\right. \\
& \times\left[2\left\langle\boldsymbol{\kappa}_{\pi}^{2}(z)\right\rangle\left(1+\frac{\left\langle\kappa_{\pi}^{2}(z)\right\rangle}{2 m_{f}^{2}}\right)\right. \\
& \left.\left.+\frac{2 \pi C_{A} A \alpha_{S}\left(\boldsymbol{k}^{2}\right)}{3\left\langle R_{c h}^{2}\right\rangle} G\left(\frac{1}{2} x_{\mathrm{P}}, \boldsymbol{k}^{2}\right)\right]\right\},
\end{aligned}
$$

where we included the effect of the longitudinal form factor. Clearly, at a sufficiently large $k$ the higher twist correction 

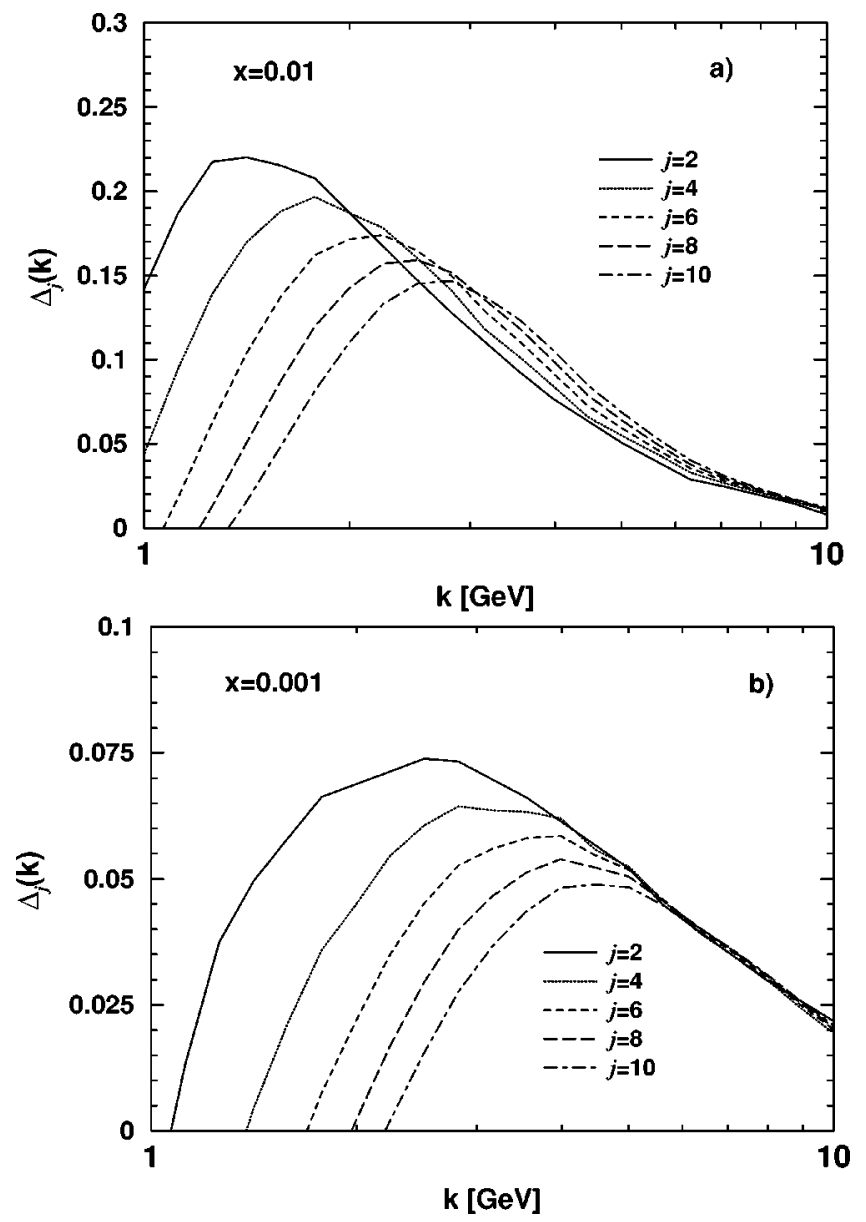

FIG. 4. Large $k$-scaling properties of the multiple convolution integrals. Shown is $\Delta_{j}(k)$ for $x=0.01$ [in panel (a)], and for $x$ $=0.001[$ panel $(\mathrm{b})]$.

will be dominated by the last term which is modelindependent, enhanced by the gluon structure function $G\left(\frac{1}{2} x_{\mathrm{P}}, \boldsymbol{k}^{2}\right)$ and rises for heavy nuclei $\propto A^{1 / 3}$.

\section{THE NUMERICAL RESULTS AND COMPARISON WITH THE E791 DATA}

\section{A. Nuclear broadening of multiple-Pomeron splitting contributions}

In the E791 kinematics $1.25 \mathrm{GeV} \leq k \leq 2.5 \mathrm{GeV}$ and $W^{2}$ $=940 \mathrm{GeV}^{2}$, so that $x_{\mathrm{P}} \sim(1-2) \times 10^{-2}$. We start with a check of the accuracy of the expansion (31) according to which

$$
\Delta_{j}(k)=\frac{1}{j-1}\left(\frac{f^{(j)}(\boldsymbol{k})}{j f^{(1)}(\boldsymbol{k})}-1\right) \approx \frac{2 \pi^{2} \delta^{2}}{3 \sigma_{0} \boldsymbol{k}^{2}} G\left(\frac{1}{2} x_{\mathrm{P}}, \boldsymbol{k}^{2}\right)
$$

must exhibit $j$-independence at sufficiently large $\boldsymbol{k}^{2}$. The results for $x_{\mathrm{P}}=2 \cdot 10^{-2}$ shown in Fig. 4(a) demonstrate this is indeed the case for very large $k \gtrsim(2-3) \mathrm{GeV}$. Slight departure from the universality can be understood in terms of the slight $k$ dependence and the broadening driven $j$ dependence of the effective exponent $\delta$. The situation at lower $k$ is a nontrivial one, because at a sufficiently small $k$ the broadening of $f^{(j)}(\boldsymbol{k})$ must be superseded by the small- $\boldsymbol{k}$ depletion, in which region

$$
\Delta_{j}(k) \sim-\frac{1}{j}
$$

The effect of depletion extends to larger $k$ with increasing $j$. Indeed, for $k=1 \mathrm{GeV}$ and $j=10$ our $\Delta_{j}(k)$ is getting close to the no-broadening estimate (39). Anyway, the finding of $\Delta_{j}(k)<0$ indicates breaking of the broadening law (30) for $j \gtrsim 5$ at $k \sim 1 \mathrm{GeV}$, the point of crossover $\Delta_{j}(k)=0$ is moving to larger $k$ with increasing $j$. The large- $k$ asymptopia is even more elusive at smaller $x$, see Fig. 4(b) for $x_{\mathrm{P}}$ $=2 \cdot 10^{-3}$. Here the broadening law (30) is only applicable at $k \gtrsim(4-5) \mathrm{GeV}$.

According to these results, the E791 range of $k$ falls in the transient region in which the higher twist expansion (31) is not applicable yet and the multiple-scattering broadening is weaker than given by Eq. (30). This suggests that in this transient region of $k$ the large- $k$ broadening of $f^{(j)}(\boldsymbol{k})$ is not sufficient for exact cancelation of shadowing effects. All numerical estimates of the A-dependence must use exact numerical results for $f^{(j)}(\boldsymbol{k})$.

\section{B. The pion wave function and $z$-distribution amplitude}

In numerical calculations of diffraction amplitudes we use a slight modification of the Jaus [21] parameterization of the pion wave function, which in our convention (10) is

$$
\psi_{\pi}(z, \boldsymbol{k}) \propto \frac{1}{z(1-z) M^{2}} \exp \left(-\frac{1}{8} R_{\pi}^{2}\left(M^{2}-4 m_{f}^{2}\right)\right)
$$

and with $R_{\pi}=2.2 \mathrm{GeV}^{-1}$ and $m_{f}=m_{u, d}=0.215 \mathrm{GeV}$ provides a consistent description of the $\pi \rightarrow \mu \nu$ decay constant $F_{\pi}$, charge radius of the pion, $\pi^{0} \rightarrow 2 \gamma$ decay rate and slope of the $\pi^{0} \rightarrow \gamma \gamma^{*}$ form factor [22] (for the related analysis see [21]). The numerical results for $\phi_{\pi}(z)$ are shown in Fig. 5 and differ only weakly from the often discussed asymptotic distribution amplitude $\phi_{\text {asym }}(z)=6 z(1-z)$ (for the review see [11-13]), in the broad range $0.2 \leq z \leq 0.8$ the difference does not exceed $10 \%$. A convenient analytic approximation to $\phi_{\pi}(z)$ given by this soft wave function is

$$
\phi_{\pi}(z)=0.6572 \log \left(1+\frac{8 z(1-z)}{R_{\pi}^{2} m_{f}^{2}}\right) \exp \left(-\frac{R_{\pi}^{2} m_{f}^{2}}{8 z(1-z)}\right)
$$

and is good to better than 1 percent apart from $z \lessgtr 0.03$ and $1-z \lesssim 0.03$

The large- $k$ asymptotics of the helicity amplitude for excitation of dijets with $\lambda+\bar{\lambda}= \pm 1$ is proportional to $\left\langle\kappa_{\pi}^{2}(z)\right\rangle$, defined in Eq. (15). The soft wave function (40) gives 


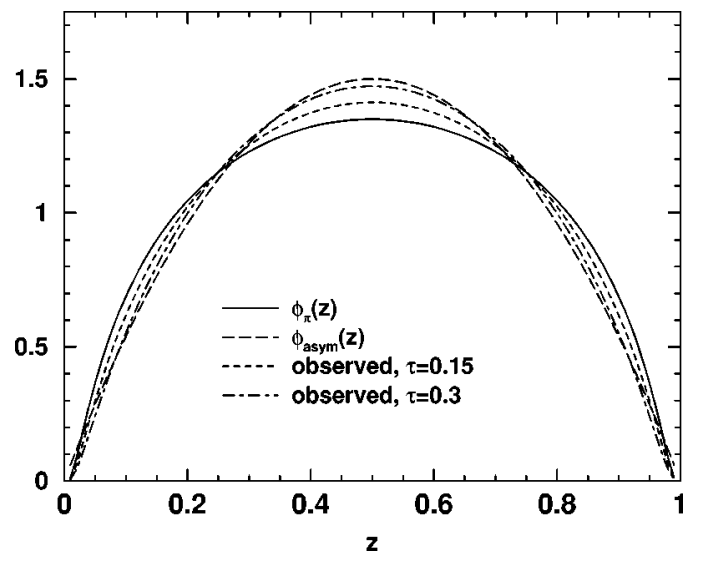

FIG. 5. The pion distribution amplitude $\phi_{\pi}(z)$. The solid line is $\phi_{\pi}(z)$ calculated from the soft wave function Eq. (40), the dashed line is the asymptotic distribution amplitude. The curves labeled "observed" show the $z$ dependence of the soft pion distribution amplitude modulated with the $z$ dependence due to the kinematical $x_{\mathrm{P}}-z$ correlation for two different values of the effective exponent $\tau$, see Eq. (43).

$$
\left\langle\kappa_{\pi}^{2}(z)\right\rangle \approx \frac{8 z(1-z)}{R^{2} \log \left(1+\frac{8 z(1-z)}{R^{2} m_{f}^{2}}\right)},
$$

i.e., $\left\langle\kappa_{\pi}^{2}(z=0.5)\right\rangle \approx 0.17 \mathrm{GeV}^{2}$, which is a natural scale for the soft pion wave function. It decreases gradually away from $z=0.5$, for instance $\left\langle\kappa_{\pi}^{2}(z=0.2)\right\rangle=\left\langle\kappa_{\pi}^{2}(z=0.8)\right\rangle$ $\approx 0.12 \mathrm{GeV}^{2}$.

Here we notice that in view of Eqs. (1) and (4) there is the kinematical $z-x_{\mathrm{P}}$ correlation $x_{\mathrm{P}} \propto[4 z(1-z)]^{-1}$. The $x_{\mathrm{P}}$ dependence of DGSF can be parametrized as $\mathcal{F}\left(\frac{1}{2} x_{\mathrm{P}}, \boldsymbol{k}^{2}\right)$ $\propto x_{\mathrm{P}}^{\tau\left(k^{2}\right)}$. With allowance for the $z-x_{\mathrm{P}}$ correlation the observed $z$-dependence of diffractive amplitudes changes from $\phi_{\pi}(z)$ to

$$
\phi_{\pi}^{o b s}(\tau, z) \propto \phi_{\pi}(z)[z(1-z)]^{\tau\left(k^{2}\right)}
$$

The phenomenological determination of the exponent $\tau\left(\boldsymbol{k}^{2}\right)$ in [10] gave $\tau \approx 0.16$ at $k=1.35 \mathrm{GeV}$ and $\tau \approx 0.22$ at $k=2$ $\mathrm{GeV}$ for the GRV-D and MRS-D parametrizations for $\mathcal{F}\left(\frac{1}{2} x_{\mathrm{P}}, \boldsymbol{k}^{2}\right)$ and $\tau \approx 0.25$ at $k=1.35 \mathrm{GeV}$ and $\boldsymbol{\tau} \approx 0.30$ at $k$ $=2 \mathrm{GeV}$ for the CTEQ-D parameterization. In Fig. 5 we show the observed z-distribution amplitudes for $\tau=0.15$ and $\tau=0.30$, evidently the observed $z$ distribution gets even closer to $\phi_{\text {asym }}(z)$.

\section{Importance of multiple Pomeron splitting processes in nuclear diffraction}

The result for the Pomeron splitting term in Eq. (32) has a deceptively simple form of the sum of the single and double scattering terms but such an interpretation would be utterly wrong. An importance of multiple nuclear rescatterings can be judged from the mean value of $j$ in expansion (29). Casting it in the form $\Phi_{0}^{(A)}(z, \boldsymbol{k})=\Sigma_{j} w_{j} f^{(j)}(\boldsymbol{k})$, for $k=2 \mathrm{GeV}$ we find

$$
\langle j\rangle=\frac{\sum_{j} w_{j} j f^{(j)}(\boldsymbol{k})}{\sum_{j} w_{j} f^{(j)}(\boldsymbol{k})}= \begin{cases}2.03 & \text { for }{ }^{12} \mathrm{C}, \\ 4.10 & \text { for }{ }^{196} \mathrm{Pt}\end{cases}
$$

which shows clearly an inadequacy of truncation of nuclear rescatterings to the single and double scattering. Indeed, at $x \sim 10^{-2}$ and $\boldsymbol{k}^{2} \sim 4 \mathrm{GeV}^{2}$ the effective shadowing cross section is quite large, $\sigma_{e f f}\left(\boldsymbol{k}^{2}\right) \sim 40 \mathrm{mb}$. Closer inspection shows that for exhausting $95 \%$ of the strength of $\Phi_{0}^{A}$ one needs the contributions up to $j=4$ for the carbon target $(\mathrm{A}=12)$ and up to $j=(8-9)$ for the platinum target $(\mathrm{A}=196)$. For the E791 energy, i.e. $x_{\mathrm{P}} \sim 10^{-2}$, this implies in conjunction with the results shown in Fig. 4 that from the viewpoint of convergence of nuclear expansions $k \gtrsim 2.5-3 \mathrm{GeV}$ are needed for the applicability of the leading plus next-to-leading expansions (35), (36), (37).

\section{Evaluation of next-to-leading twist corrections}

The next-to-leading twist correction can conveniently by parameterized as $H / k^{2}$. First we evaluate the contribution to $H$ coming from the pion wave function. It is controlled by the moment $\left\langle\kappa_{\pi}^{2}(z)\right\rangle$. For the $0.2 \lesssim z \lesssim 0.8$ which are relevant to the E791 data, we take in further estimates $\left\langle\kappa_{\pi}^{2}(z)\right\rangle$ $=0.15 \mathrm{GeV}^{2}$. We notice that numerically $\left\langle\kappa^{2}(z=0.5)\right\rangle$ $\approx 3.5 m_{f}^{2}$, which entails that on the free nucleon target nextto-leading twist effects come predominantly from the helicity component $\propto \boldsymbol{\Phi}_{1}^{2}$ in the cross section, see Eq. (17):

$$
H_{\pi}^{(1)}=\frac{\delta^{2}\left\langle\kappa_{\pi}^{2}(z)\right\rangle^{2}}{m_{f}^{2}} \approx 0.55 \delta^{2} \mathrm{GeV}^{-2} \sim 2.2 \mathrm{GeV}^{-2} .
$$

Here we took $\delta^{2} \sim 4$ appropriate for $k \gtrsim(2.5-3) \mathrm{GeV}$ where the asymptotic expansion (37) is applicable.

A similar estimate for the contribution to $H$ from from the helicity component $\propto \Phi_{0}^{2}$ in the cross section is

$$
H_{\pi}^{(0)} \sim 1.3 \mathrm{GeV}^{-2} \text {. }
$$

For $x_{\mathrm{P}} \sim 10^{-2}$ and $\boldsymbol{k}^{2} \sim(3-4) \mathrm{GeV}^{2}$ relevant to the E791 kinematics, the analysis [10] gives $\alpha_{S} G\left(\frac{1}{2} x_{\mathrm{P}}, \boldsymbol{k}^{2}\right) \approx 1-1.2$. Then the nuclear rescattering or broadening contribution to next-to-leading twist is (we use the nuclear density parameters from the compilation [29])

$$
\begin{aligned}
& H_{A}=\frac{4 \pi \delta^{2} C_{A} A^{2}}{3\left\langle R_{c h}^{2}\right\rangle} \alpha_{S}\left(\boldsymbol{k}^{2}\right) G\left(\frac{1}{2} x_{\mathrm{P}}, k^{2}\right) \approx(0.16-0.2) \delta^{2} A^{1 / 3} \\
& \sim\left\{\begin{array}{cc}
1.5 \mathrm{GeV}^{2} & \text { for }{ }^{12} \mathrm{C}, \\
3.5 \mathrm{GeV}^{2} & \text { for }{ }^{196} \mathrm{Pt} .
\end{array}\right.
\end{aligned}
$$

Lumping together all three contributions, we find $H^{P t} \sim 7$ $\mathrm{GeV}^{2}$ and $H^{C} \sim 5 \mathrm{GeV}^{2}$. Consequently, no simple expansion in the leading and next-to-leading contributions is possible in the E791 region of jet momentum and an accurate numerical evaluation of broadening of $f^{(j)}(\boldsymbol{k})$ is called upon. 


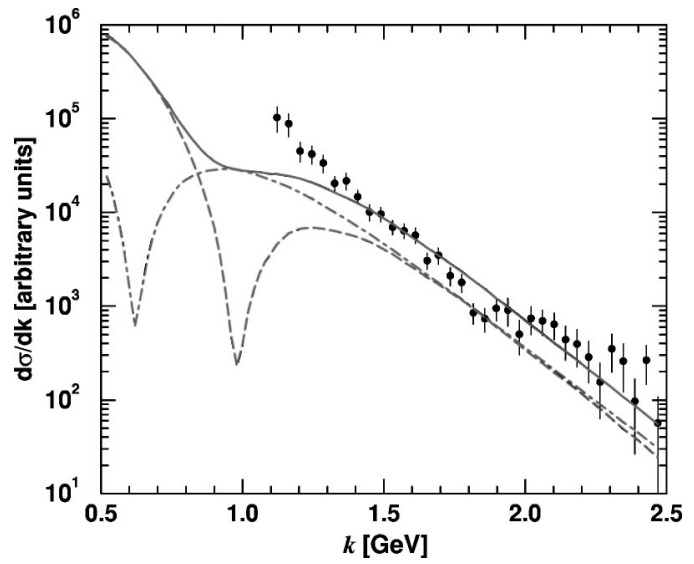

FIG. 6. The E791 data [9] for the differential diffractive dijet cross section $d \sigma / d k$ for the ${ }^{196} \mathrm{Pt}$ target with the theoretical calculations. The data are not normalized. The dash-dotted line shows the contribution of the helicity amplitude $\Phi_{0}^{(A)}$; the dashed line is the contribution from $\boldsymbol{\Phi}_{1}^{(A)}$. The solid line is the total result.

\section{E. Comparison with the E791 data: $k^{2}$ distributions}

In Fig. 6 we show our numerical results for the $\Delta^{2}$-integrated nuclear diffraction cross section for the platinum target. We are interested in the large $\boldsymbol{k}$ where the contributions $\propto \psi_{\pi}(z, \boldsymbol{k})$ died out, which in both helicity amplitudes is preceded by the zero. In order to have a crude idea on where this happens, we stretched our calculations for platinum target down to $k=0.5 \mathrm{GeV}$. The amplitude $\Phi_{0}$ has a zero at $k \sim 0.65 \mathrm{GeV}$. Because the term $\propto \psi_{\pi}(z, \boldsymbol{k})$ is sensitive to nuclear shadowing and to the soft cross section $\sigma_{0}$ thereof, the position of the zero is model-dependent. In $\boldsymbol{\Phi}_{1}$ the second component is of higher twist and $\boldsymbol{\Phi}_{1}$ has a zero at larger $k \sim 1 \mathrm{GeV}$. The impact of these zeros of diffractive amplitudes is manifest up to $k \gtrsim 1.5 \mathrm{GeV}$, which is still another reason why a comparison of our predictions with the experimental data is justified only at $k \gtrsim 1.5 \mathrm{GeV}$.

The E791 data give only the $k$ dependence of the acceptance-corrected cross section without absolute normalization. The normalization of our theoretical curve is the eyeball fit to the data, the agreement with the experimentally observed $k$-dependence is good at $k \gtrsim 1.5 \mathrm{GeV}$. In the theoretical calculations we include consistently the $\boldsymbol{k}^{2}-x_{\mathrm{P}}$ correlation discussed in Sec. II following Eq. (17).
At E791 energy our leading twist theoretical cross section would have followed the law $k^{-n}$ with the slope

$$
n \approx 4+2 \delta+2 \tau\left(\boldsymbol{k}^{2}\right) \approx 8.7-8.8 .
$$

Our numerical results do not exhibit simple dependence $\propto k^{-n}$, if we define the local slope $n$ as

$$
n=-\frac{\partial \log \sigma_{D}}{\partial \log \boldsymbol{k}^{2}},
$$

then for $k \gtrsim 2.5 \mathrm{GeV}$ we find $n \sim 12$, whereas around $k=2$ $\mathrm{GeV}$ the slope $n \approx 10$. We attribute these large values of local $n$ to very large higher twist corrections.

In Fig. 6 and Fig. 7 we show separately the contributions to the diffractive cross section of the two helicity states: the leading plus next-to-leading twist component with $\lambda+\bar{\lambda}=0$ and the higher twist component $\lambda+\bar{\lambda}= \pm 1$. They are of comparable magnitude, the higher twist component $\propto \boldsymbol{\Phi}_{1}^{2}$ starts dying out and the component $\propto \Phi_{0}^{2}$ starts taking over only at $k \gtrsim 2.5-3 \mathrm{GeV}$, in perfect agreement with evaluation of higher twists in Sec. IV D.

Figure 7 shows our predictions for the future experimental tests of the absolute normalization of diffractive cross section. In all the numerical calculations we used the parametrization labeled 'D-GRV', from [10]. Here for the sake of convenience we plot $k^{8} d \sigma_{D} /\left.d z d k^{2}\right|_{z=0.5}$. As an illustration of the energy dependence of dijet production here we also show the predictions for $E_{\pi}=5 \mathrm{TeV}$. In this case $x_{\mathrm{P}} \sim 10^{-3}$ and $\delta \sim 1.7$, so that in the leading twist we expect $n \sim 8$. Furthermore, because of the lower value of $\delta$ the higher twist effects would be about half of those for $E_{\pi}=500 \mathrm{GeV}$ in the E791 experiment, cf. Fig. 4(a) and 4(b). Indeed, for $E_{\pi}=5$ $\mathrm{TeV} k^{8} d \sigma_{D} /\left.d z d k^{2}\right|_{z=0.5}$ exhibits much weaker dependence on $\boldsymbol{k}^{2}$.

\section{F. Comparison with the E791 data: nuclear mass number dependence}

The $A$-dependence of nuclear cross sections is often parameterized as $\sigma \propto A^{\alpha}$. The E791 experiment uses the carbon and platinum targets and defines the exponent $\alpha$ as

$$
\alpha=\frac{\log \frac{\sigma_{1}}{\sigma_{2}}}{\log \frac{A_{1}}{A_{2}}} .
$$
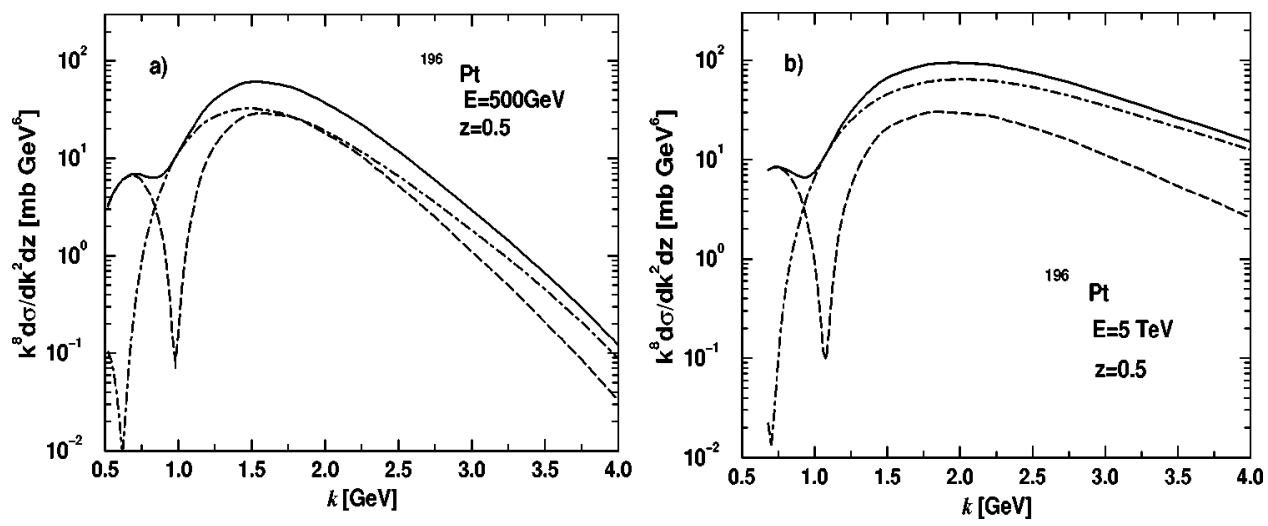

FIG. 7. Theoretical predictions for the differential dijet-cross section for the ${ }^{196} \mathrm{Pt}$ target. Panel (a) is for the energy of the E791 experiment, $E=500 \mathrm{GeV}$; panel (b) for $E=5 \mathrm{TeV}$. The dashdotted line shows the contribution of the helicity amplitude $\Phi_{0}^{(A)}$; the dashed line is the contribution from $\boldsymbol{\Phi}_{1}^{(A)}$. The solid line is the total result. 


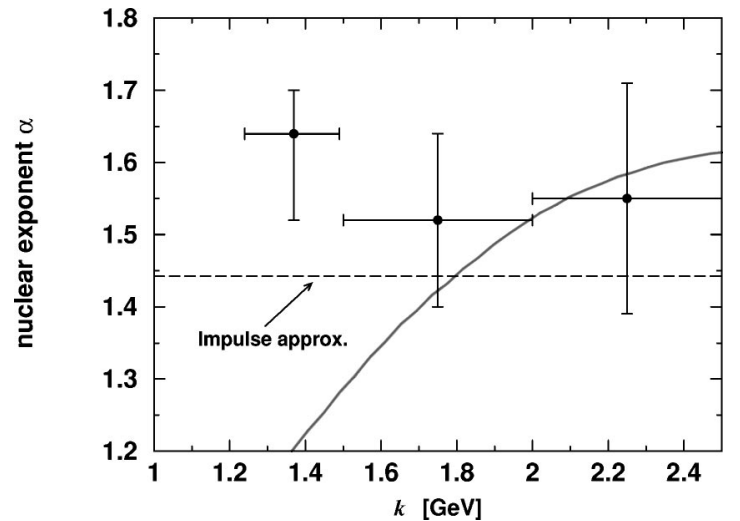

FIG. 8. Exponent $\alpha$ of the atomic mass number dependence of the dijet cross section with the results from E791 [9]. The dashed line shows the impulse approximation result; the solid line is the result of the full multiple scattering series.

For the reference, in the impulse approximation

$$
\sigma \propto \frac{A^{2}}{\left\langle R_{c h}^{2}\right\rangle}
$$

and simple evaluation for Pt and $\mathrm{C}$ nuclei gives $\alpha_{I A}=1.44$ (the average slope for the $A$ dependence in the same interval gives $\alpha_{I A}=1.39$, for instance, see [30]). A comparison of our numerical results for $\mathrm{Pt}$ and $\mathrm{C}$ nuclei yields the $k$-dependence of the exponent $\alpha$ shown in Fig. 8.

As we discussed in Sec. IV A, at $k \leqq 1.5 \mathrm{GeV}$ antishadowing/broadening effects are too weak to cancel nuclear shadowing, which explains the small value of $\alpha\left(\boldsymbol{k}^{2}\right)<\alpha_{I A}$. On the other hand, at larger $k$ higher-twist corrections become substantial and give $\alpha\left(\boldsymbol{k}^{2}\right)>\alpha_{I A}$ at $k \gtrsim 1.8$ $\mathrm{GeV}$. At large $\boldsymbol{k}^{2}$ we find again good agreement with the E791 results.

\section{G. Comparison with the E791 data: $z$-distributions and the pion wave function}

To leading twist diffraction of pions into dijets uniquely allows to measure the pion distribution amplitude $\phi_{\pi}(z)$. As we have seen above, for moderately large $k$ studied experimentally higher twist corrections are very large. The parameter $\left\langle\kappa_{\pi}^{2}(z)\right\rangle$ of higher twist correction from the pion wave function varies with $z$ and is a model dependent one, our estimate (42) gives only a crude idea on its $z$ dependence and Eqs. (45), (46) must be regarded as numerical estimates within the factor two. Notwithstanding these uncertainties, even on the free nucleon target one must be able to distinguish experimentally between the double-humped CZ [11] and asymptotic distribution functions.
To the contrary, the nuclear-rescattering driven higher twist correction is model-independent. The $x_{\mathrm{P}}-z$ correlation driven $z$ dependence of $G\left(\frac{1}{2} x_{\mathrm{P}}, \boldsymbol{k}^{2}\right)$ is very weak, it only slightly enhances the cross section around $z=\frac{1}{2}$. For this reason, even if higher-twist dominated, the diffraction off heavy targets is a good probe of the pion distribution amplitude $\phi_{\pi}(z)$. The E791 paper does not give the acceptancecorrected $z$-distributions. Ashery concludes [9] that within the $\sim 20 \%$ experimental error bars the observed E791 $z$-distribution is consistent with the Monte Carlo modeling based on the asymptotic pion distribution $\phi_{\text {asym }}(z)$. Because the observed $z$-distribution (41) given by our model soft wave function is very close to the asymptotic one, it is perfectly consistent with the E791 data.

\section{CONCLUSIONS}

We developed the perturbative QCD description of diffraction dissociation of pions into hard dijets on nucleons and nuclei. To leading twist dijet excitation is shown to be dominated by the Pomeron splitting mechanism and the two diffractive helicity amplitudes are shown to be proportional to the unintegrated gluon structure function of the proton. We derived an multiple-Pomeron splitting expansion of nuclear amplitudes which is of antishadowing nature. To leading twist there is a remarkable cancelation of nuclear attenuation and antishadowing or broadening effects. We obtained a model-independent estimate for next-to-leading twist corrections driven by nuclear rescatterings. These higher twist corrections are shown to be very large up to jet momenta $\boldsymbol{k}^{2} \geq(5-7) \mathrm{GeV}^{2}$ and affect substantially the $\boldsymbol{k}^{2}$ and atomic mass number of the diffraction cross section. The model dependence of extraction of the $z$ dependence of the pion distribution amplitude is shown to be weak for diffraction off heavy nuclei. Our calculations based on the recent determination of unintegrated gluon structure function of the proton reproduce well the basic experimental findings from the recent E791 experiment. A simple interpretation of the observed $k$ dependence is not possible though because in the E791 range of $k$ the cross section is overwhelmed by higher twist effects.

\section{ACKNOWLEDGMENTS}

We are grateful to Danny Ashery for helpful correspondence on the E791 data. Thanks are due to B.G. Zakharov for discussions during the early stages of this work and to D. Ivanov for comments. This research has partly been supported by the grant INTAS 97-30494.
[1] L.D. Landau and I.Ya. Pomeranchuk, Zh. Eksp. Teor. Fiz. 24, 505 (1953).

[2] I.Ya. Pomeranchuk and E.L. Feinberg, Dokl. Akad. Nauk SSSR 93, 439 (1953); E.L. Feinberg, Zh. Éksp. Teor. Fiz. 1, 177 (1955) [Sov. Phys. JETP 28, 242 (1955)]; E.L. Feinberg and I.Ya. Pomeranchuk, Nuovo Cimento, Suppl. 4, 652 (1956); A.I. Akhiezer and A.G. Sitenko, Zh. Éksp. Teor. Fiz. 5, 652 (1957) [Sov. Phys. JETP 32, 794 (1957)]; 33, 1040 (1957).

[3] R.J. Glauber, Phys. Rev. 99, 1515 (1955). 
[4] N.N. Nikolaev and B.G. Zakharov, Z. Phys. C 53, 331 (1992).

[5] N.N. Nikolaev and B.G. Zakharov, Phys. Lett. B 332, 177 (1994).

[6] M. Genovese, N.N. Nikolaev, and B.G. Zakharov, Phys. Lett. B 378, 347 (1996).

[7] K. Golec-Biernat, J. Kwiecinski, and A.D. Martin, Phys. Rev. D 58, 094001 (1998).

[8] A.V. Radyushkin, Phys. Lett. B 385, 333 (1996).

[9] D. Ashery, Invited talk at X International Light-Cone Meeting, Heidelberg, 2000, hep-ex/0008036.

[10] I.P. Ivanov and N.N. Nikolaev, hep-ph/0004206.

[11] V.L. Chernyak and A.R. Zhitnitsky, Phys. Rep. 112, 173 (1984).

[12] R. Jakob and P. Kroll, Phys. Lett. B 315, 463 (1993); 319, 545 (1993); P. Kroll and M. Raulfs, Phys. Lett B 387, 848 (1996) and references therein.

[13] G.P. Lepage and S.J. Brodsky, Phys. Rev. D 22, 2157 (1980); S.J. Brodsky, H.-C. Pauli, and S.S. Pinsky, Phys. Rep. 301, 299 (1998).

[14] L.L. Frankfurt, G.A. Miller, and M.I. Strikman, Phys. Lett. B 304, 1 (1993).

[15] R.J. Glauber, in Lectures in Theoretical Physics, edited by W. Brittain and L.G. Dunham (Interscience, New York, 1959), Vol. 1; R.J. Glauber and G. Matthiae, Nucl. Phys. B21, 135 (1970).

[16] N.N. Nikolaev, A.V. Pronyaev, and B.G. Zakharov, Phys. Rev. D 59, 091501 (1999).

[17] C. Daum et al., Nucl. Phys. B182, 269 (1981); Phys. Lett. 89B, 281 (1980).
[18] G. Bellini et al., Phys. Rev. Lett. 48, 1697 (1982).

[19] M. Zielinski et al., Phys. Rev. D 30, 1855 (1984); Z. Phys. C 16, 197 (1983).

[20] J. Nemchik, N.N. Nikolaev and B.G. Zakharov, in Workshop on CEBAF at Higher Energies, 1994, edited by N. Isgur and P. Stoler (CEBAF, Newport News, VA, 1994), pp. 415-464, nucl-th/9406005.

[21] W. Jaus, Phys. Rev. D 44, 2851 (1991).

[22] G. Schwiete, Diploma thesis, University of Bonn, forthcoming.

[23] N.N. Nikolaev, A.V. Pronyaev, and B.G. Zakharov, Pis'ma Zh. Éksp. Teor. Fiz. 68, 604 (1998) [JETP Lett. 68, 634 (1998)].

[24] Particle Data Group, C. Caso et al., Eur. Phys. J. C 3, 1 (1998).

[25] N.N. Nikolaev and B.G. Zakharov, Z. Phys. C 49, 607 (1991).

[26] B.G. Zakharov, Yad. Fiz. 61, 924 (1998) [Phys. At. Nucl. 61, 838 (1998)].

[27] W. Schäfer, Unitarity driven nonperturbative effects in deep inelastic scattering, Berichte des Forschungszentrum Jülich 3170 (Forschungszentrum Jülich, Jülich, 1999); Talk given at 8th International Workshop on Deep Inelastic Scattering and QCD (DIS 2000), Liverpool, England, 2000, hep-ph/0006263.

[28] V.N. Gribov, Zh. Éksp. Teor. Fiz. 56, 892 (1969) [Sov. Phys. JETP 29, 483 (1969)].

[29] H. de Vries, C.W. de Jaeger, and C. de Vries, At. Data Nucl. Data Tables 36, 496 (1987).

[30] B.Z. Kopeliovich, J. Nemchik, N.N. Nikolaev, and B.G. Zakharov, Phys. Lett. B 324, 469 (1994). 